\title{
Quantitative EEG as a biomarker in mild cognitive impairment with Lewy bodies
}

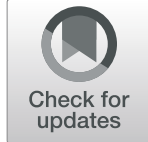

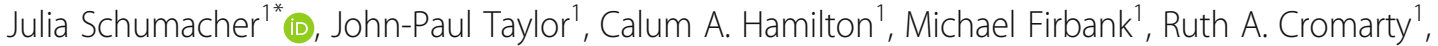 \\ Paul C. Donaghy ${ }^{1}$, Gemma Roberts ${ }^{1}$, Louise Allan ${ }^{1,3}$, Jim Lloyd ${ }^{2}$, Rory Durcan ${ }^{1}$, Nicola Barnett ${ }^{1}$, John T. O'Brien ${ }^{4}$ and \\ Alan J. Thomas ${ }^{1}$
}

\begin{abstract}
Objectives: To investigate using quantitative EEG the (1) differences between patients with mild cognitive impairment with Lewy bodies (MCl-LB) and MCl with Alzheimer's disease (MCl-AD) and (2) its utility as a potential biomarker for early differential diagnosis.

Methods: We analyzed eyes-closed, resting-state, high-density EEG data from highly phenotyped participants (39 $\mathrm{MCl}-\mathrm{LB}, 36 \mathrm{MCl}-\mathrm{AD}$, and 31 healthy controls). EEG measures included spectral power in different frequency bands (delta, theta, pre-alpha, alpha, and beta), theta/alpha ratio, dominant frequency, and dominant frequency variability. Receiver operating characteristic (ROC) analyses were performed to assess diagnostic accuracy.

Results: There was a shift in power from beta and alpha frequency bands towards slower frequencies in the prealpha and theta range in MCI-LB compared to healthy controls. Additionally, the dominant frequency was slower in MCl-LB compared to controls. We found significantly increased pre-alpha power, decreased beta power, and slower dominant frequency in MCI-LB compared to MCI-AD. EEG abnormalities were more apparent in MCI-LB cases with more diagnostic features. There were no significant differences between MCI-AD and controls. In the ROC analysis to distinguish MCI-LB from MCI-AD, beta power and dominant frequency showed the highest area under the curve values of 0.71 and 0.70 , respectively. While specificity was high for some measures (up to 0.97 for alpha power and 0.94 for theta/alpha ratio), sensitivity was generally much lower.
\end{abstract}

Conclusions: Early EEG slowing is a specific feature of MCI-LB compared to MCI-AD. However, there is an overlap between the two $\mathrm{MCl}$ groups which makes it difficult to distinguish between them based on EEG alone.

Keywords: Dementia with Lewy bodies, Alzheimer's disease, Quantitative electroencephalography, Biomarker

\section{Background}

The early diagnosis of dementia is becoming increasingly important as it is likely that potential disease-modifying treatments will have their greatest effect at this stage. Additionally, accurate and early differential diagnosis of dementia subtypes is crucial for correct patient

\footnotetext{
* Correspondence: julia.schumacher@newcastle.ac.uk

${ }^{1}$ Translational and Clinical Research Institute, Faculty of Medical Sciences, Newcastle University, Biomedical Research Building 3rd floor, Campus for Ageing and Vitality, Newcastle upon Tyne NE4 5PL, UK

Full list of author information is available at the end of the article
}

stratification for clinical trials and research studies and for optimizing clinical care such as by highlighting the need to carefully avoid using medication with anticholinergic properties and encouraging active identification of therapeutic targets such as orthostatic hypotension and constipation [1].

The intermediate stage between normal aging and dementia in which cognitive decline is present, but independence in activities of daily living is still preserved, is referred to as mild cognitive impairment (MCI) [2, 3]. While much research has focused on MCI patients who

C C The Author(s). 2020 Open Access This article is licensed under a Creative Commons Attribution 4.0 International License, which permits use, sharing, adaptation, distribution and reproduction in any medium or format, as long as you give appropriate credit to the original author(s) and the source, provide a link to the Creative Commons licence, and indicate if changes were made. The images or other third party material in this article are included in the article's Creative Commons licence, unless indicated otherwise in a credit line to the material. If material is not included in the article's Creative Commons licence and your intended use is not permitted by statutory regulation or exceeds the permitted use, you will need to obtain permission directly from the copyright holder. To view a copy of this licence, visit http://creativecommons.org/licenses/by/4.0/ The Creative Commons Public Domain Dedication waiver (http://creativecommons.org/publicdomain/zero/1.0/) applies to the data made available in this article, unless otherwise stated in a credit line to the data. 
later develop Alzheimer's disease (AD), $\mathrm{MCI}$ in the context of dementia with Lewy bodies (DLB) has only been characterized more recently with research criteria recently published [4-6]. Differential diagnosis is often impeded by a substantial clinical overlap between $A D$ and DLB, which is especially pronounced in early stages, highlighting the need for objective biomarkers. In DLB, dopaminergic brain imaging and myocardial scintigraphy are indicative biomarkers [7]; these can improve diagnostic accuracy at the MCI stage [8] and are included in the new research criteria for MCI with Lewy bodies (MCI-LB). EEG has been suggested as a non-invasive alternative, and an abnormal EEG is listed as a supportive biomarker in the DLB diagnostic criteria [7]. However, its value for differential diagnosis at the MCI stage is less certain.

The aims of the present study were therefore to investigate changes in quantitative EEG measures in a large cohort of well-characterized patients, all of whom had biomarker assessments with both dopaminergic and cardiac MIGB imaging, and to test the utility of EEG as a biomarker for early differential diagnosis.

The secondary aim was to better understand the relationship between EEG abnormalities in the MCI-LB group and the core Lewy body symptoms. We hypothesized that EEG abnormalities would be more severe in patients with more core symptoms.

\section{Methods}

\section{Participants}

All participants in this study were over 60 years of age. Patients with a clinical diagnosis of MCI in memory services in the north east of England were screened and approached for this study if they reported additional clinical symptoms suggestive of Lewy body disease (e.g., mood changes, sleep disturbances, or autonomic symptoms) or any core DLB features (visual hallucinations, cognitive fluctuations, parkinsonism, and REM sleep behavior disorder). After obtaining written informed consent, participants underwent a baseline clinical assessment including the Addenbrooke's Cognitive Examination-Revised (ACE-R), from which the Mini-Mental State Examination (MMSE) score was derived; the Unified Parkinson's Disease Rating Scale motor sub-score (UPDRS-III); the Epworth Sleepiness Scale (ESS); and the Geriatric Depression Scale (GDS). Additionally, the Instrumental Activities of Daily Living (IADL) Scale, the Clinician Assessment of Fluctuations (CAF), the Dementia Cognitive Fluctuations Scale (DCFS), the North-East Visual Hallucinations Interview (NEVHI), the Neuropsychiatric Inventory (NPI), and the Mayo Sleep Questionnaire (MSQ) were administered to informants, and the Clinical Dementia Rating (CDR) Scale and the Cumulative Illness Rating Scale for Geriatrics (CIRS-G) were completed based on the clinical history and research assessments. In addition to a detailed clinical assessment, participants had already undergone dopaminergic imaging with ${ }^{123} \mathrm{I}-\mathrm{N}$ fluoropropyl-2 $\beta$-carbomethoxy-3 $\beta$-(4-iodophenyl) singlephoton emission computed tomography (FP-CIT SPECT) and ${ }^{123}$ iodine-metaiodobenzylguanidine (MIBG) myocardial scintigraphy through their involvement in an ongoing study investigating the diagnostic accuracy of imaging biomarkers in MCI, and this information was used to apply diagnostic criteria (see below).

After the initial baseline visit, participants were followed annually for up to 3 years with a mean follow-up time of 16.5 months (standard deviation $=6.5$ months). Patients who were taking dopaminergic medication were assessed in the "ON" motor state.

\section{Diagnosis}

Diagnoses were based on all information available at the end of the recruitment period (December 2019) including any baseline and follow-up visits. MCI diagnoses were made independently by a consensus panel of three experienced old-age psychiatrists (AJT, PCD, JPT) in accordance with NIA-AA criteria [3], i.e., subjective and objective cognitive impairment with maintained independence of function with minimal aids or assistance and a CDR of 0 or 0.5. Patients with a diagnosis of dementia were excluded from the study. Furthermore, patients with possible contributing frontotemporal or vascular etiologies or with a history of parkinsonism of more than 1 year prior to the onset of cognitive impairment were excluded. The presence or absence of the core Lewy body symptoms was rated by the panel utilizing the rating scales and all information from the clinical assessments [7]. Findings from the FP-CIT and MIBG scans were used for diagnosis (see below), but the clinical MCI diagnoses as well as the rating of the presence/ absence of core DLB symptoms were performed blind to these imaging findings.

A diagnosis of MCI with probable Alzheimer's disease (MCI-AD) was given to patients who had no core Lewy body symptoms, negative FP-CIT and MIBG findings, and evidence of cognitive decline that was characteristic of $\mathrm{AD}$, i.e., they met the additional NIA-AA criterion for "etiology of MCI consistent with $\mathrm{AD}$ pathophysiologic process" [3].

Probable MCI with Lewy bodies (MCI-LB) was diagnosed if a patient had two or more core Lewy body symptoms or one core symptom in addition to a positive FP-CIT or MIBG scan [6].

Out of 103 MCI participants who were included in the study, 20 had only one core Lewy body symptom with negative FP-CIT and MIBG scans or no core symptoms in addition to a positive FP-CIT or MIBG scan, i.e., they did not meet the criteria for either MCI-AD or probable 


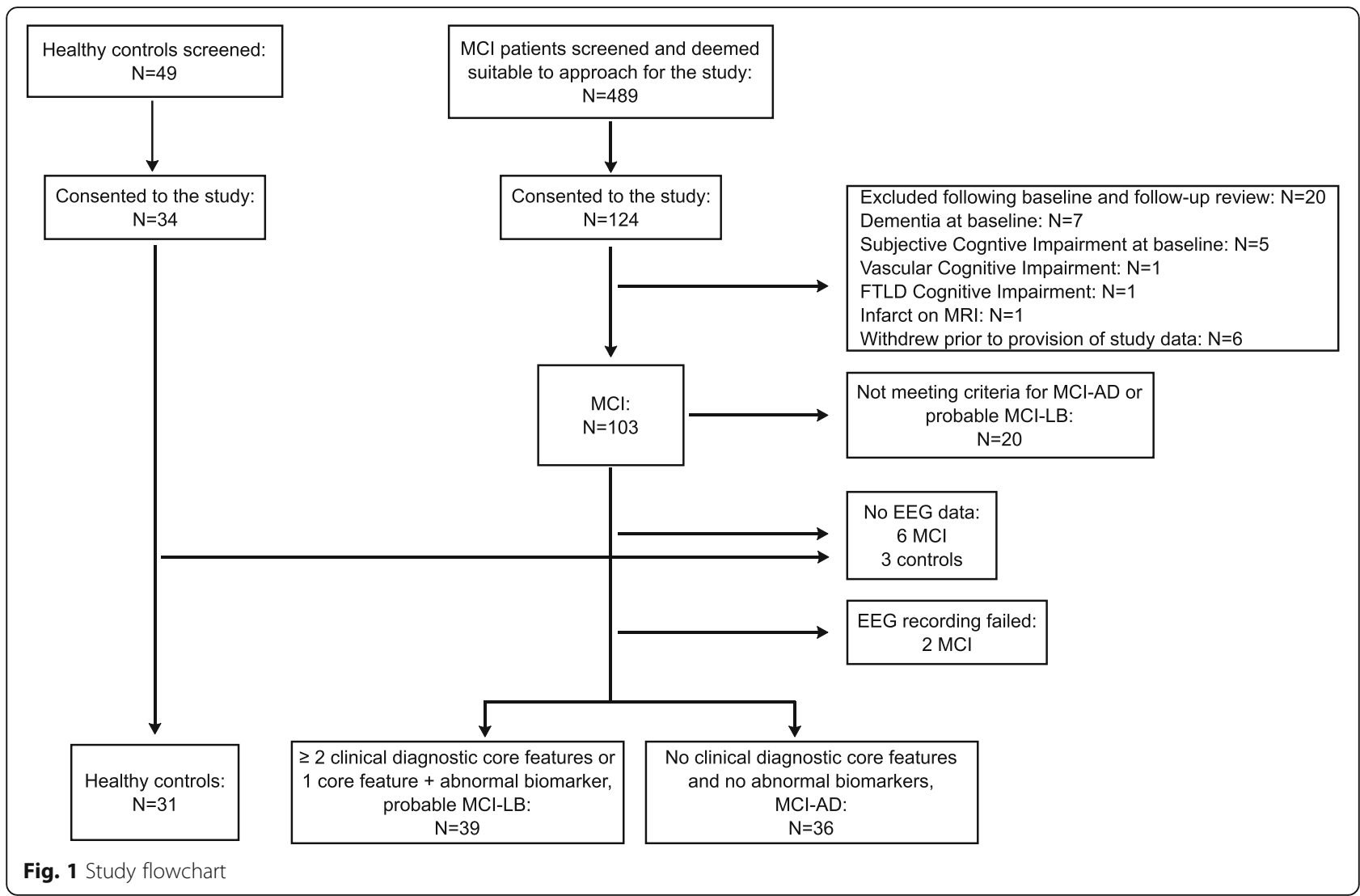

MCI-LB and were therefore not included in the present analysis (Fig. 1). Additionally, eight MCI participants did not have usable EEG data available. This study therefore included 39 participants who were diagnosed with probable MCI-LB and 36 who were diagnosed with MCI-AD. Healthy control participants $(N=31)$ were recruited from relatives and friends of patients and from a local research register. Control participants had the same assessment as the patients and had no history of psychiatric or neurological illness and no evidence of any cognitive decline. They also had normal structural MR imaging. The study was approved by the local ethics committee, and written informed consent was obtained from all participants.

\section{EEG acquisition and pre-processing}

Resting-state high-density EEG recordings were acquired from all participants using Waveguard caps (ANT Neuro, The Netherlands) comprising 128 sintered Ag/ $\mathrm{AgCl}$ electrodes that were placed according to the 10-5 system. Participants were seated during the recording and instructed to remain awake. Electrode impedance was kept below $5 \mathrm{k} \Omega$, and continuous EEG data were recorded at a sampling frequency of $1024 \mathrm{~Hz}$. Three hundred seconds of eyes-closed data were recorded from each participant. Participants were supervised by the
EEG technician during the recording to monitor adherence to the protocol. The ground electrode was attached to the right clavicle, and all EEG channels were referenced to Fz during recording.

Pre-processing of eyes-closed EEG data was performed using the EEGLAB toolbox (version 14) in MATLAB (R2017a) [9] and was blinded to group membership. First, EEG data were bandpass-filtered between 0.3 and $54 \mathrm{~Hz}$ using a second-order Butterworth filter and split into non-overlapping epochs of $2 \mathrm{~s}$. Subsequently, EEG recordings were visually inspected to identify noisy channels and noisy epochs which were excluded prior to applying independent component analysis for further artifact removal. The resulting components were visually inspected, and components representing muscular, cardiac, ocular, or electrical $(50 \mathrm{~Hz}$ line noise) artifacts were rejected. The previously excluded channels were then replaced using spherical spline interpolation, and data were recomputed against the average reference. For each participant, the first 45 2-s-long artifact-free epochs were selected for further analysis.

\section{Frequency analysis}

For each 2-s epoch, the power spectral density (PSD) was estimated using Bartlett's method in MATLAB (R2017a) with a frequency resolution of $0.5 \mathrm{~Hz}$ and a 
Hamming window across the power spectrum from 2 to $30 \mathrm{~Hz}$, for each electrode separately. To compensate for inter-individual variability in brain neurophysiology, anatomy, and physical tissue properties, the PSD was normalized by the total power across the power spectrum [10].

For each electrode separately, the mean power across all included epochs was estimated for different standard EEG frequency bands including delta $(2-4 \mathrm{~Hz})$, theta (4-5.5 $\mathrm{Hz})$, pre-alpha $(5.5-8 \mathrm{~Hz})$, alpha $(8-13 \mathrm{~Hz})$, and beta $(13-$ $30 \mathrm{~Hz}$ ). Higher frequencies were excluded because these are particularly affected by muscular artifacts [11]. The dominant frequency was calculated as the frequency with the highest power between 4 and $15 \mathrm{~Hz}$ (averaged across epochs). Dominant frequency was calculated for all electrodes as well as from occipital electrodes only (PO9, PO7, POO9h, PO5, O1, PO3, POO3h, OI1h, POz, Oz, PO4, POO4h, PO6, O2, OI2h, PO8, POO10h, PO10). Dominant frequency variability was defined as the standard deviation of dominant frequency across epochs [12].

\section{Statistics}

Statistical analyses were performed in SPSS and R (https://www.r-project.org/). Relative power within the different frequency bands was compared between the three groups using a multivariate ANOVA with a within-subject factor of frequency band and a betweensubject factor of diagnosis, followed by univariate ANOVAs and post hoc tests, Bonferroni-corrected for multiple comparisons. Since relative power was not normally distributed in all groups, the variables were log-transformed before applying the ANOVA. Theta/ alpha ratio, dominant frequency, and dominant frequency variability were compared between the groups using univariate ANOVAs followed by post hoc tests (Bonferroni-corrected). To account for differences in the number of male and female participants in the three groups, sex was included as a covariate in all analyses.

To test the applicability of this analysis to a clinical setting, the group comparison of relative power within the different frequency bands was repeated only including the 21 electrodes that are part of the 10-20 system that is routinely used in clinical practice.

Additionally, to test whether EEG changes have a different spatial distribution in the different groups, we conducted a supplementary analysis splitting the whole set of electrodes into four macroscopic regions (frontal, central, lateral, and posterior) and applying a repeated measures ANOVA with region as the within-subject factor and diagnosis as the between-subject factor [10]. If the interaction between region and diagnosis was significant, this was followed up by post hoc univariate ANOVAs.

A receiver operating characteristic (ROC) analysis was conducted in $\mathrm{R}$ to assess the sensitivity and specificity of the different EEG measures to distinguish between MCI$\mathrm{AD}$ and MCI-LB patients. The sensitivity/specificity cutoff was determined using Youden's index.

To assess the association between the range of Lewy body symptomatology and EEG abnormalities, we performed an exploratory analysis in which we compared quantitative EEG measures between MCI-LB patients who had two core symptoms or one core symptom and one abnormal biomarker $(N=13)$ with those patients who had more than two core symptoms/abnormal biomarkers $(N=26)$ using two-sample $t$ tests. Furthermore, Spearman's correlations between symptom/biomarker count (ranging from 2 to 6 ) and the different EEG measures were computed in the MCI-LB group.

The association between quantitative EEG measures and overall cognitive impairment was assessed using Spearman's correlations, in the MCI-AD and MCI-LB groups separately. $p$ values were FDR-corrected for multiple comparisons.

Additionally, we investigated the association between EEG characteristics and the core Lewy body symptoms of visual hallucinations and cognitive fluctuations which have been shown to be related to EEG abnormalities in dementia patients $[10,13,14]$. To this end, two-sample $t$ tests were performed, dichotomizing the MCI-LB group according to the presence/absence of visual hallucinations and cognitive fluctuations.

Given previous reports of an effect of acetylcholinesterase inhibitors on the EEG signal [15, 16], a twosample $t$ test was performed in the MCI-LB group to compare the EEG characteristics between patients who were taking acetylcholinesterase inhibitors and those patients not taking these medications.

\section{Results}

\section{Demographics}

All groups were similar in age (Table 1). The proportion of male participants was higher in the MCI-LB group whereas more MCI-AD patients were female. MCI-LB patients had significantly lower years of education compared to controls, but there was no significant difference between the two MCI groups in terms of education. More MCI-LB patients were taking cholinesterase inhibitors and Parkinson's disease medication compared to MCI-AD patients. The two MCI groups were matched in terms of overall cognitive impairment. As expected, the MCI-LB group had more parkinsonism, higher cognitive fluctuation, and visual hallucination scores compared to the MCI-AD group.

\section{EEG frequency analysis}

The multivariate ANOVA revealed an overall effect of diagnosis: $F(10,196)=5.1, p<0.001$. Follow-up univariate ANOVAs showed the following (see Table 2 and 
Table 1 Demographic and clinical variables, mean (standard deviation)

\begin{tabular}{|c|c|c|c|c|}
\hline & $\mathrm{HC}(\mathrm{N}=31)$ & $\mathrm{MCl}-\mathrm{AD}(\mathrm{N}=36)$ & $\mathrm{MCl}-\mathrm{LB}(\mathrm{N}=39)$ & Group differences \\
\hline Male to female & $22: 9$ & $15: 21$ & $35: 4$ & $\begin{array}{l}x^{2}=20.0, p<0.001^{a} \\
p(\mathrm{HC}, \mathrm{MCl}-\mathrm{AD})=0.02 \\
p(\mathrm{HC}, \mathrm{MCl}-\mathrm{LB})=0.045 \\
p(\mathrm{MCl}-\mathrm{AD}, \mathrm{MCl}-\mathrm{LB})<0.001\end{array}$ \\
\hline Age & $73.7(7.3)$ & $76.1(7.7)$ & $74.7(6.4)$ & $F(2,103)=1.0, p=0.38^{b}$ \\
\hline AChEl & - & $7(19 \%)^{\mathrm{e}}$ & $18(46 \%)^{f}$ & $x^{2}=5.7, p=0.02^{c}$ \\
\hline PD meds & - & $0^{\mathrm{e}}$ & $4(10 \%)^{f}$ & $x^{2}=3.8, p=0.052^{c}$ \\
\hline Years of education & $14.7(4.0)^{9}$ & $12.9(3.4)^{h}$ & $12.1(2.8)$ & $\begin{array}{l}F(2,100)=5.1, p=0.008^{C} \\
p(\mathrm{HC}, \mathrm{MCl}-\mathrm{AD})=0.12 \\
p(\mathrm{HC}, \mathrm{MCl}-\mathrm{LB})=0.006 \\
p(\mathrm{MCl}-\mathrm{AD}, \mathrm{MCl}-\mathrm{LB})=0.87\end{array}$ \\
\hline ACE-R & $92.7(4.2)$ & $82.4(8.5)$ & $83.8(9.2)$ & $t_{73}=0.7, p=0.50^{d}$ \\
\hline MMSE & $28.5(1.1)$ & $26.9(2.1)$ & $26.6(2.5)$ & $t_{73}=0.7, p=0.51^{d}$ \\
\hline UPDRS III & $5.5(4.4)$ & $15.1(13.8)$ & $23.3(14.2)$ & $t_{73}=2.5, p=0.01^{d}$ \\
\hline DCFS & - & $6.9(1.9)^{i}$ & $8.5(3.3)^{k}$ & $t_{61}=2.4, p=0.02^{d}$ \\
\hline CAF total & - & $1.4(2.7)^{i}$ & $3.7(4.2)^{k}$ & $t_{61}=2.5, p=0.02^{d}$ \\
\hline NPI total & - & $8.6(9.3)^{i}$ & $16.4(12.9)^{k}$ & $t_{61}=2.7, p=0.01^{d}$ \\
\hline NEVHI & - & $0.8(1.5)^{\mathrm{e}}$ & $2.7(4.1)$ & $t_{71}=2.6, p=0.01^{d}$ \\
\hline GDS & $1.3(1.8)$ & $3.5(2.5)$ & $5.1(4.1)$ & $\begin{array}{l}F(2,103)=13.4, p<0.001^{b} \\
p(\mathrm{HC}, \mathrm{MCl}-\mathrm{AD})=0.01 \\
p(\mathrm{HC}, \mathrm{MCl}-\mathrm{LB})<0.001 \\
p(\mathrm{MCl}-\mathrm{AD}, \mathrm{MCl}-\mathrm{LB})=0.08\end{array}$ \\
\hline
\end{tabular}

$A C E-R$ Addenbrooke's Cognitive Examination-Revised, $A C h E l$ number of patients taking acetylcholinesterase inhibitors, $C A F$ total clinician assessment of fluctuation total score, DCFS Dementia Cognitive Fluctuation Scale, GDS Geriatric Depression Scale, $H C$ healthy controls, MCI-AD mild cognitive impairment with Alzheimer's disease, MCI-LB probable mild cognitive impairment with Lewy bodies, MMSE Mini-Mental State Examination, NEVHI North-East Visual Hallucinations Interview, NPI Neuropsychiatric Inventory, PD meds number of patients taking dopaminergic medication for the management of Parkinson's disease symptoms, UPDRS III Unified Parkinson's Disease Rating Scale III (motor subsection)

${ }^{a}$ Chi-square test $\mathrm{HC}, \mathrm{MCl}-\mathrm{AD}, \mathrm{MCl}-\mathrm{LB}$

bUnivariate ANOVA HC, MCI-AD, MCI-LB

${ }^{\mathrm{C} C h i-s q u a r e}$ test MCI-AD, MCI-LB

${ }^{\mathrm{d}}$ Student's $t$ test MCI-AD, MCI-LB

${ }^{\mathrm{e}} \mathrm{N}=34$

${ }^{\mathrm{f}} N=38$

${ }^{9} N=29$

${ }^{\mathrm{h}} N=35$

${ }^{i} N=27$

${ }^{\mathrm{k}} N=36$

Figs. 2 and 3): There were no group differences in terms of delta power. Theta power was increased in MCI-LB (mean $=8.8$, standard deviation $(\mathrm{SD})=4.8)$ compared to controls (mean $=5.7, \mathrm{SD}=3.2$ ) with no significant difference between controls and MCI-AD (mean $=7.0, \mathrm{SD}=$ 3.1) or between the two MCI groups. Pre-alpha power was increased in MCI-LB (mean $=28.8, S D=14.2)$ and MCI-AD (mean $=19.6, \mathrm{SD}=10.2)$ compared to controls (mean $=13.3, \mathrm{SD}=9.2$ ), and it was further increased in MCI-LB compared to MCI-AD. Alpha power was decreased in MCI-LB (mean $=28.3, \mathrm{SD}=14.7)$ compared to controls (mean $=39.9, \mathrm{SD}=15.8)$ with no difference between controls and MCI-AD (mean = 35.6, SD = 12.7) or between the two MCI groups. However, when the alpha band was split further into low-alpha $(8-10 \mathrm{~Hz})$ and high-alpha $(10-13 \mathrm{~Hz})$, it became evident that highalpha power was significantly reduced in MCI-LB compared to both controls $(p<0.001)$ and MCI-AD $(p<$
$0.001)$ with no difference between MCI-AD and controls $(p=1.0)$ while there were no group differences in terms of low-alpha power $(F(2,102)=1.7, p=0.2)$. Beta power was reduced in MCI-LB (mean $=18.9, \mathrm{SD}=11.0)$ compared to both controls (mean $=26.7, \mathrm{SD}=10.3)$ and MCI-AD (mean $=25.4, \mathrm{SD}=8.7)$, but not different between MCI-AD and controls.

The theta/alpha ratio was increased in MCI-LB (mean $=0.51, \mathrm{SD}=0.21)$ compared to controls (mean $=$ $0.34, \mathrm{SD}=0.17$ ), but not significantly different between MCI-AD (mean $=0.42, \mathrm{SD}=0.16$ ) and controls or between the two MCI groups.

Dominant frequency was slower in MCI-LB (mean = $7.2, \mathrm{SD}=1.1$ ) compared to both $\mathrm{MCI}-\mathrm{AD}$ (mean $=8.0$, $\mathrm{SD}=1.1$ ) and controls (mean $=8.4, \mathrm{SD}=1.2$ ), but there was no significant difference between controls and MCIAD. These results did not change when calculating the dominant frequency from the occipital electrodes instead 
Table 2 Group comparison of quantitative EEG characteristics

\begin{tabular}{|c|c|c|c|c|}
\hline & $\mathrm{HC}$ & $\mathrm{MCl}-\mathrm{AD}$ & MCl-LB & Group comparison \\
\hline Delta power & $14.1[11.3,17.0]$ & $14.1[11.8,16.3]$ & $14.9[12.7,17.1]$ & $F(2,102)=0.8, p=0.47$ \\
\hline \multirow[t]{2}{*}{ Theta power } & $5.7[4.6,6.9]$ & $7.0[6.0,8.1]$ & $8.8[7.2,10.3]$ & $F(2,102)=4.8, p=0.01$ \\
\hline & & & & $\begin{array}{l}p(\mathrm{HC}, \mathrm{MCl}-\mathrm{AD})=0.19 \\
p(\mathrm{HC}, \mathrm{MCl}-\mathrm{LB})=0.004 \\
p(\mathrm{MCl}-\mathrm{AD}, \mathrm{MCl}-\mathrm{LB})=0.46\end{array}$ \\
\hline \multirow[t]{2}{*}{ Pre-alpha power } & $13.3[9.9,16.7]$ & $19.6[16.1,23.1]$ & $28.8[24.2,33.4]$ & $F(2,102)=16.0, p<0.001$ \\
\hline & & & & $\begin{array}{l}p(\mathrm{HC}, \mathrm{MCl}-\mathrm{AD})=0.006 \\
p(\mathrm{HC}, \mathrm{MCl}-\mathrm{LB})<0.001 \\
p(\mathrm{MCl}-\mathrm{AD}, \mathrm{MCl}-\mathrm{LB})=0.02\end{array}$ \\
\hline \multirow[t]{2}{*}{ Alpha power } & $39.9[34.1,45.6]$ & $33.6[29.3,37.9]$ & $28.3[23.6,33.1]$ & $F(2,102)=5.5, p=0.005$ \\
\hline & & & & $\begin{array}{l}p(\mathrm{HC}, \mathrm{MCl}-\mathrm{AD})=0.39 \\
p(\mathrm{HC}, \mathrm{MCl}-\mathrm{LB})=0.001 \\
p(\mathrm{MCl}-\mathrm{AD}, \mathrm{MCl}-\mathrm{LB})=0.07\end{array}$ \\
\hline \multirow[t]{2}{*}{ Beta power } & $26.7[22.9,30.4]$ & $25.4[22.4,28.4]$ & $18.9[15.3,22.5]$ & $F(2,102)=8.1, p=0.001$ \\
\hline & & & & $\begin{array}{l}p(\mathrm{HC}, \mathrm{MCl}-\mathrm{AD})=1.0 \\
p(\mathrm{HC}, \mathrm{MCl}-\mathrm{LB})<0.001 \\
p(\mathrm{MCl}-\mathrm{AD}, \mathrm{MCl}-\mathrm{LB})=0.001\end{array}$ \\
\hline \multirow[t]{2}{*}{ Theta/alpha ratio } & $0.34[0.27,0.40]$ & $0.42[0.36,0.47]$ & $0.51[0.44,0.57]$ & $F(2,102)=7.5, p<0.001$ \\
\hline & & & & $\begin{array}{l}p(\mathrm{HC}, \mathrm{MCl}-\mathrm{AD})=0.22 \\
p(\mathrm{HC}, \mathrm{MCl}-\mathrm{LB})=0.001 \\
p(\mathrm{MCl}-\mathrm{AD}, \mathrm{MCl}-\mathrm{LB})=0.10\end{array}$ \\
\hline \multirow[t]{2}{*}{ DF, all electrodes } & $8.4[8.0,8.9]$ & $8.0[7.6,8.3]$ & $7.2[6.8,7.6]$ & $F(2,102)=8.7, p<0.001$ \\
\hline & & & & $\begin{array}{l}p(\mathrm{HC}, \mathrm{MCl}-\mathrm{AD})=0.29 \\
p(\mathrm{HC}, \mathrm{MCl}-\mathrm{LB})<0.001 \\
p(\mathrm{MCl}-\mathrm{AD}, \mathrm{MCl}-\mathrm{LB})=0.01\end{array}$ \\
\hline \multirow[t]{2}{*}{ DF, occipital electrodes } & $8.5[8.1,9.0]$ & $8.0[7.6,8.3]$ & $7.3[6.9,7.7]$ & $F(2,102)=8.7, p<0.001$ \\
\hline & & & & $\begin{array}{l}p(\mathrm{HC}, \mathrm{MCl}-\mathrm{AD})=0.16 \\
p(\mathrm{HC}, \mathrm{MCl}-\mathrm{LB})<0.001 \\
p(\mathrm{MCl}-\mathrm{AD}, \mathrm{MCl}-\mathrm{LB})=0.03\end{array}$ \\
\hline \multirow[t]{2}{*}{ DFV, occipital electrodes } & $1.3[0.9,1.6]$ & $1.4[1.1,1.7]$ & $1.0[0.8,1.2]$ & $F(2,102)=3.6, p=0.03$ \\
\hline & & & & $\begin{array}{l}p(\mathrm{HC}, \mathrm{MCl}-\mathrm{AD})=0.54 \\
p(\mathrm{HC}, \mathrm{MCl}-\mathrm{LB})=1.0 \\
p(\mathrm{MCl}-\mathrm{AD}, \mathrm{MCl}-\mathrm{LB})=0.17\end{array}$ \\
\hline
\end{tabular}

Mean [95\% confidence interval] of different quantitative EEG characteristics. Group comparisons were performed using univariate ANOVAs followed by post hoc tests, Bonferroni-corrected for multiple comparisons. Sex was included as a covariate

$D F$ dominant frequency, $D F V$ dominant frequency variability, $H C$ healthy controls, $M C I-A D$ mild cognitive impairment with Alzheimer's disease, $M C l-L B$ probable mild cognitive impairment with Lewy bodies

of using all electrodes. Dominant frequency variability was not significantly different between the groups.

The results did not change when restricting the analysis to the 21 electrodes that are part of the 10-20 system (see Supplementary Table S1).

The regional analysis showed very similar results compared to the analysis where all electrodes were combined (see Supplementary Table S2). The only slight difference was that alpha power was significantly reduced in MCI-LB compared to MCI-AD patients in posterior $(p=0.047)$ and lateral $(p=0.01)$ regions whereas mean alpha power from all electrodes was not significantly different between the two MCI groups $(p=0.07)$.

\section{ROC analysis for diagnostic discrimination}

Area under the ROC curve (AUC) values, sensitivity, and specificity for the different EEG measures for differentiating between MCI-LB and MCI-AD are shown in Table 3. Beta power and dominant frequency achieved the highest AUC values of 0.71 and 0.70, respectively. Specificity was high for some measures (up to 0.97 for alpha power); however, sensitivity was generally much lower.

\section{Association with clinical symptoms}

There was a significant reduction in alpha power in MCI-LB patients with visual hallucinations $(N=9$, mean $(\mathrm{SD})=19.1(6.4))$ compared to those without visual 


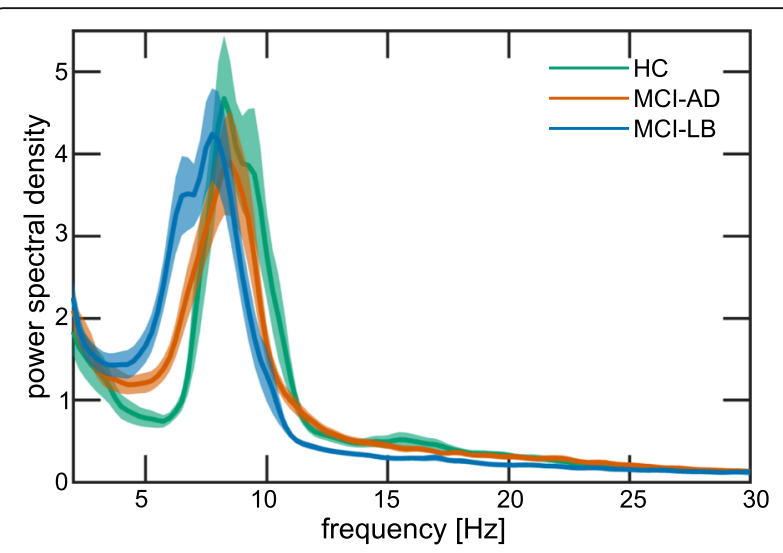

Fig. 2 Mean power spectra for the three diagnostic groups. Shaded areas indicate standard errors. $\mathrm{HC}$, healthy controls; $\mathrm{MCl}-\mathrm{AD}$, mild cognitive impairment with Alzheimer's disease; MCl-LB, probable mild cognitive impairment with Lewy bodies

hallucinations $\left(N=30\right.$, mean $(\mathrm{SD})=31.1(15.4) ; t_{37}=2.5$, $p=0.02$; Fig. 4a). Additionally, the theta/alpha ratio was increased in patients with visual hallucinations (mean $(\mathrm{SD})=0.61(0.13))$ compared to patients without visual hallucinations (mean $(\mathrm{SD})=0.47(0.22), t_{24}=2.4, p=$ 0.02, Fig. 4b).

None of the EEG measures showed a significant difference between those MCI-LB patients with cognitive fluctuations $(N=21)$ compared to those without cognitive fluctuations $(N=18)$ or between MCI-LB patients taking cholinesterase inhibitors $(N=18)$ compared to those patients not taking cholinesterase inhibitors $(N=20$, all $p>0.1)$. Similarly, EEG measures were not different between MCI-AD patients taking cholinesterase inhibitors $(N=7)$ compared to those MCI-AD patients not taking these medications $(N=27)$.

MCI-LB patients with more than two core symptoms/ abnormal biomarkers had significantly higher pre-alpha power, lower alpha power, and a higher theta/alpha ratio compared to MCI-LB patients with only two core symptoms or one core symptom and one abnormal biomarker (Supplementary Table S3). Higher delta power, theta power, pre-alpha power, and theta/alpha ratio correlated with higher Lewy body symptom/biomarker count whereas higher alpha power, beta power, and dominant frequency correlated with lower Lewy body symptom/ biomarker count (Supplementary Table S3). There were no significant differences in overall cognition (ACE- $R$ scores) between MCI-LB patients with more than two core symptoms/abnormal biomarkers compared to patients with two symptoms, and the Lewy body symptom/ biomarker count was not significantly correlated with the ACE-R score (see Supplementary Table S3).

More severe cognitive impairment (as measured by the ACE-R) was correlated with higher pre-alpha power, lower alpha power, and decreased dominant frequency in the MCI-AD group (Supplementary Table S4). In the MCI-LB group, more severe cognitive impairment correlated with higher theta power, higher pre-alpha power, lower alpha power, higher theta/alpha ratio, and decreased dominant frequency (Supplementary Table S4).

\section{Discussion}

In this study, we investigated the differences in quantitative EEG measures between highly phenotyped patients with MCI-AD and MCI-LB and in comparison with similarly aged healthy controls. We showed that there are significant differences between the two MCI groups at the group level with more severe EEG abnormalities in MCI-LB compared to MCI-AD and that these EEG changes correlated with the burden of Lewy body features, supporting their high specificity for Lewy body disease. However, their diagnostic accuracy for differentiating MCI-LB from MCI-AD is modest and not consistent with previous studies [17].

Overall, the findings suggest a slowing of the EEG in MCI-LB patients compared to healthy controls by a shift in power from beta and alpha frequency bands towards slower frequencies in the pre-alpha and theta range. This was also reflected by a shift of the dominant frequency towards slower frequencies. These findings are consistent with the results from previous EEG studies at the dementia stage which generally report an increase in slowwave activity and slowing of the dominant EEG rhythm in DLB [10, 18-20]. A slowing of the dominant frequency has also been reported in MCI-LB patients before [21].

We did not observe any group differences in dominant frequency variability which has previously been found to be increased in DLB patients compared to controls [12, $17,19]$. However, other studies have failed to replicate these findings $[10,18,22]$, and our results further indicate that an increase in dominant frequency variability may not be a reliable feature of Lewy body disease.

The only difference between MCI-AD patients and healthy controls was an increase in pre-alpha power, suggesting only a small degree of EEG slowing. This is again consistent with previous reports at the dementia stage which have generally found less severe EEG abnormalities in AD compared to DLB [10, 12, 19]. Additionally, we found significantly increased pre-alpha power, decreased beta power, and slower dominant frequency in the MCI-LB compared to the MCI-AD group, which indicates more severe EEG slowing in MCI-LB than in $\mathrm{MCI}-\mathrm{AD}$ patients and mirrors the findings in dementia patients. Furthermore, we found more severe EEG abnormalities in MCI-LB patients with more core Lewy body symptoms and/or abnormal biomarkers and a higher symptom count correlated with more severe EEG slowing. This indicates that there is an association 

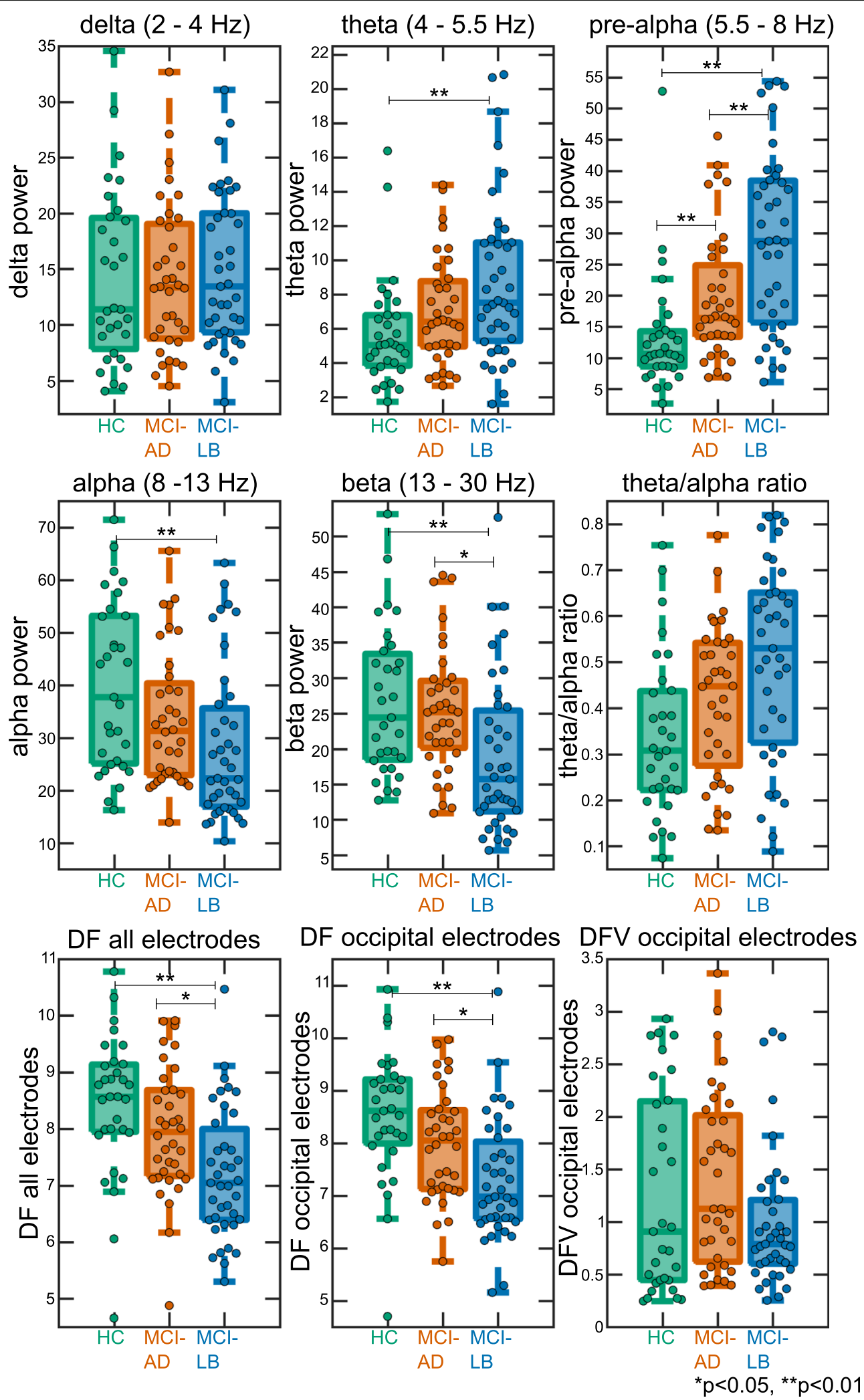

Fig. 3 (See legend on next page.) 
(See figure on previous page.)

Fig. 3 Group comparison of quantitative EEG characteristics. In each boxplot, the central line corresponds to the sample median; the upper and lower border of the box represent the 25th and 75th percentile, respectively; and the length of the whiskers is 1.5 times the interquartile range. Corresponding results from statistical comparisons between the groups are presented in Table 2. DF, dominant frequency; DFV, dominant frequency variability; $\mathrm{HC}$, healthy controls; $\mathrm{MCl}-\mathrm{AD}$, mild cognitive impairment with Alzheimer's disease; MCI-LB, probable mild cognitive impairment with Lewy bodies

between more widespread Lewy body disease and more severe EEG abnormalities and suggests that Lewy body disease might affect electro-cortical activity in a dosedependent manner.

While there were significant group-level differences in EEG characteristics between the two MCI groups, it is difficult to translate this, at present, into a measure for use in early clinical diagnosis. Power in the beta frequency range and dominant frequency had the highest diagnostic accuracy; however, they only reached moderate AUC values of around 0.7. The specificity for MCILB was very high for certain EEG measures (up to 0.97 for alpha power with a cutoff of $<20.5$ ) which suggests that if a substantial shift of power towards slower frequencies is observed, i.e., in the case of a very abnormal EEG, a diagnosis of MCI-LB over MCI-AD becomes highly likely. The relationship between EEG slowing and Lewy body symptom count (see above) suggests that these might be the MCI-LB patients with more widespread Lewy body disease and these are easier to distinguish from MCI-AD patients based on their more severe EEG slowing. However, sensitivity was generally much lower, i.e., in the case of a more normal EEG, differentiating between MCI-AD and MCI-LB is difficult and many MCI-LB cases would be missed by applying these measures for diagnostic purposes. These findings thus show that early changes in quantitative EEG characteristics are specific, but not sensitive to Lewy body disease which is in alignment with the diagnostic performance of other biomarkers such as FP-CIT and MIBG in early Lewy body disease [8].
In a previous EEG study in MCI patients, Bonanni et al. [17] found that all MCI patients who converted to DLB within a 3-year follow-up period had an abnormal EEG whereas $93 \%$ of $\mathrm{MCI}$ patients who developed $\mathrm{AD}$ within the follow-up period had normal EEGs. The high specificity of EEG abnormalities for MCI-LB in this report is in line with our findings whereas the high sensitivity stands in contrast to the findings of the present study. However, the sample in this previous study was more selective by only including patients who developed dementia within 3 years and might therefore be biased towards MCI patients with more severe disease and thus having a rapid progression to dementia. When also taking into account those MCI patients who did not convert to dementia within 3 years, diagnostic accuracy was reduced to an overall predictive value of $76 \%$ [17]. Furthermore, in a multi-center study in dementia patients, the diagnostic accuracy of EEG was also found to be much lower [19], indicating that heterogeneity in EEG acquisition and analysis protocols across different centers might be an important limiting factor.

Another previous EEG study that attempted to differentiate MCI-AD from MCI-LB did not find any EEG measures that reliably distinguished between the two MCI subgroups [21]. However, this was a retrospective study of selected MCI-AD and MCI-LB patients who were recruited using clinical criteria which differed across the several centers who supplied patient data. Furthermore, participants from the different centers were also assessed using different EEG protocols which again makes the comparison difficult [21].

Table 3 Results from receiver operating characteristic (ROC) analysis to distinguish MCl-AD from MCl-LB

\begin{tabular}{|c|c|c|c|c|}
\hline EEG measure & $\mathrm{AUC}[95 \% \mathrm{Cl}]$ & Cutoff for MCI-LB & Sensitivity & Specificity \\
\hline Delta power & $0.54[0.41,0.67]$ & $>21.9$ & 0.23 & 0.89 \\
\hline Theta power & $0.60[0.47,0.73]$ & $>10.7$ & 0.33 & 0.89 \\
\hline Pre-alpha power & $0.68[0.56,0.81]$ & $>28.1$ & 0.56 & 0.83 \\
\hline Alpha power & $0.66[0.53,0.78]$ & $<20.5$ & 0.41 & 0.97 \\
\hline Beta power & $0.71[0.59,0.83]$ & $<19.0$ & 0.61 & 0.81 \\
\hline Theta/alpha ratio & $0.64[0.51,0.77]$ & $>0.56$ & 0.49 & 0.83 \\
\hline DF, all electrodes & $0.70[0.58,0.82]$ & $<7.1$ & 0.51 & 0.86 \\
\hline DF, occipital electrodes & $0.69[0.57,0.81]$ & $<7.1$ & 0.51 & 0.86 \\
\hline
\end{tabular}

The sensitivity/specificity cutoff was determined using Youden's index

$A U C$ area under the receiver operating curve, $C I$ confidence interval, $D F$ dominant frequency, $M C l-A D$ mild cognitive impairment with Alzheimer's disease, $M C l-L B$ probable mild cognitive impairment with Lewy bodies 

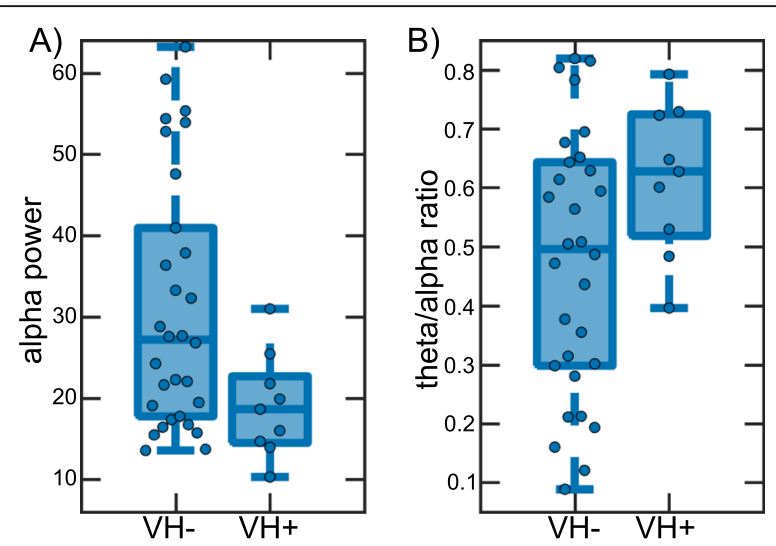

Fig. 4 Comparison of quantitative EEG characteristics between patients with and without visual hallucinations. a Comparison of alpha power and $\mathbf{b}$ theta/alpha ratio between $\mathrm{MCl}-\mathrm{LB}$ patients with visual hallucinations $(\mathrm{VH}+, \mathrm{N}=9)$ and without visual hallucinations $(\mathrm{VH}-, \mathrm{N}=30)$. In each boxplot, the central line corresponds to the sample median; the upper and lower border of the box represent the 25th and 75th percentile, respectively; and the length of the whiskers is 1.5 times the interquartile range

We explored the influence of the presence of visual hallucinations on quantitative EEG characteristics in MCI-LB patients and found that early EEG slowing, both in terms of a reduction of power in the alpha frequency range and a shift of power towards slower frequencies, might be more severe in patients who experience visual hallucinations compared to those patients who do not have visual hallucinations, a finding which has previously been reported in dementia patients [14]. However, the number of patients experiencing visual hallucinations in our cohort was small $(N=9)$ and these findings should therefore be interpreted with caution, and replication in a larger group of patients with a higher occurrence of visual hallucinations is needed.

\section{Limitations}

Strengths of the present study include consistent and robust clinical assessments, diagnoses by a consensus panel, the use of two diagnostic biomarkers, and the prospective longitudinal design of the study. A potential limitation is the use of acetylcholinesterase inhibitors in the MCI patients as more patients in the MCI-LB group were taking these medications compared to the MCI-AD group. In the north east of England, services implement expert advice and thus have high prescription rates for DLB/MCI-LB patients because neuropsychiatric symptoms are treated using anti-dementia drugs early on. It has been shown that cholinergic medication can normalize EEG measures [15, 16], and it is therefore possible that differences between the two MCI groups were occluded by the higher number of MCI-LB patients taking these medications. However, when comparing
MCI-LB patients who were taking acetylcholinesterase inhibitors to those who were not, we did not find any significant differences in any EEG measures. The same was found in the MCI-AD patients although this result should be interpreted with caution because of the small number of MCI-AD patients who were taking acetylcholinesterase inhibitors $(N=7)$. Furthermore, it would be unethical to withdraw medication, and including medicated patients is more reflective of clinical practice. Another potential limitation is the lack of $\mathrm{AD}$ biomarkers in our study.

\section{Conclusions}

In conclusion, the present study suggests that early EEG slowing is specific to MCI-LB and, from a diagnostic point of view, a very abnormal EEG favors MCI-LB over MCI-AD. However, the overlap between the two MCI groups is large, and for patients with a more normal EEG, it is difficult to distinguish between the two MCI groups based on EEG characteristics alone. Given its specificity for Lewy body disease burden, EEG may be a promising future diagnostic biomarker and may have value as part of a panel of other biomarkers. To investigate this further, there needs to be a consensus regarding the standardization of acquisition protocols, analysis approaches, and choice of EEG parameters. Work in this field will undoubtedly yield benefits similar to those seen with CSF biomarkers for example [23]. More complex EEG analyses such as functional connectivity or source reconstruction methods may provide better sensitivity for detecting differences between the two MCI groups as well as between MCI-AD patients and controls [21, 24]. Furthermore, multimodal approaches including EEG features in addition to MIBG/FP-CIT or structural MRI markers may be another route by which it will be possible to achieve better accuracy for the early distinction between $\mathrm{AD}$ and DLB at the MCI stage.

\section{Supplementary information}

Supplementary information accompanies this paper at https://doi.org/10. 1186/s13195-020-00650-1.

Additional file 1: Table S1. Group comparison of quantitative EEG characteristics, restricting the analysis to 21 electrodes from the 10-20 system. Table S2. Group comparison of quantitative EEG characteristics, splitting the set of electrodes into four macroscopic regions. Table S3. Association between Lewy body symptom severity and EEG characteristics in the MCl-LB group. Two-sample t-tests comparing EEG measures between $\mathrm{MCl}$-LB patients with two symptoms/biomarkers $(N=13)$ and $\mathrm{MCl}-\mathrm{LB}$ patients with more than two symptoms/biomarkers $(N=26)$ and Spearman's correlations between EEG characteristics and symptom/biomarker count (ranging from 2 to 6). P-values are FDR-corrected for multiple comparisons. Table S4. Association between the severity of overall cognitive impairment (ACE-R scores) and EEG characteristics in the $\mathrm{MCl}$ $\mathrm{LB}$ and $\mathrm{MCl}-\mathrm{AD}$ groups using Spearman's correlations. P-values are FDRcorrected for multiple comparisons. 


\section{Abbreviations}

ACE-R: Addenbrooke's Cognitive Examination-Revised; AD: Alzheimer's disease; AUC: Area under the ROC curve; CAF: Clinician assessment of fluctuations; CDR: Clinical Dementia Rating; CIRS-G: Cumulative IIIness Rating Scale for Geriatrics; DCFS: Dementia Cognitive Fluctuations Scale; DLB: Dementia with Lewy bodies; EEG: Electroencephalography; ESS: Epworth Sleepiness Scale; FDR: False discovery rate; FP-CIT: ${ }^{123} \mathrm{I}-\mathrm{N}$ -

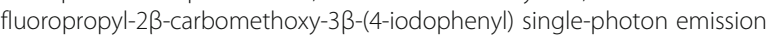
computed tomography; GDS: Geriatric Depression Scale; IADL: Instrumental Activities of Daily Living; MCI-AD: Mild cognitive impairment with Alzheimer's disease; MCI-LB: Mild cognitive impairment with Lewy bodies; MIBG: ${ }^{123}$ Iodine-metaiodobenzylguanidine myocardial scintigraphy; MSQ: Mayo Sleep Questionnaire; MMSE: Mini-Mental State Examination; NEVHI: North-East Visual Hallucinations Interview; NPI: Neuropsychiatric Inventory; PSD: Power spectral density; ROC: Receiver operating characteristic; UPDRS-III: Unified Parkinson's Disease Rating Scale motor subscore

\section{Acknowledgements}

Not applicable

\section{Authors' contributions}

JS analyzed and interpreted the data, performed the statistical analyses, and wrote the manuscript. CAH, MF, RAC, GR, RD, and NB acquired the data and revised the manuscript. PCD acquired the data, diagnosed the patients, and revised the manuscript. MF, LA, JL, and JTOB conceived the study, participated in its design and coordination, and revised the manuscript. JPT and AJT conceived the study, participated in its design and coordination, diagnosed the patients, interpreted the data, and revised the manuscript. All authors read and approved the final version of the manuscript.

\section{Funding}

This research was funded by the Alzheimer's Research UK (ARUK-PG2015-13) and supported by the NIHR Newcastle Biomedical Research Centre (grant numbers BH120812 and BH120878). GE Healthcare provided the FP-CIT radioligand for this investigator-led study.

\section{Availability of data and materials}

The data that support the findings of this study are available from the corresponding author, upon reasonable request.

\section{Ethics approval and consent to participate}

The study was approved by the Newcastle \& North Tyneside 2 Research Ethics Committee (reference number: 15/NE/0420). Written informed consent was obtained from all participants prior to study participation.

\section{Consent for publication}

Not applicable

\section{Competing interests}

The authors declare that they have no competing interests.

\section{Author details}

'Translational and Clinical Research Institute, Faculty of Medical Sciences, Newcastle University, Biomedical Research Building 3rd floor, Campus for Ageing and Vitality, Newcastle upon Tyne NE4 5PL, UK. ${ }^{2}$ Nuclear Medicine Department, Newcastle upon Tyne Hospitals NHS Foundation Trust, Newcastle upon Tyne, UK. ${ }^{3}$ Institute of Health Research, University of Exeter, Exeter, UK. ${ }^{4}$ Department of Psychiatry, University of Cambridge School of Medicine, Cambridge CB2 OSP, UK.

\section{Received: 13 May 2020 Accepted: 2 July 2020}

\section{Published online: 08 July 2020}

\section{References}

1. Taylor J-P, McKeith IG, Burn DJ, Boeve BF, Weintraub D, Bamford C, et al. New evidence on the management of Lewy body dementia. Lancet Neurol. 2020:19:157-69. https://doi.org/10.1016/S1474-4422(19)30153-X

2. Petersen RC, Smith GE, Waring SC, Ivnik RJ, Tangalos EG, Kokmen E. Mild cognitive impairment. Arch Neurol. 1999:56:303 Available from: http:// archneur.jamanetwork.com/article.aspx?doi=10.1001/archneur.56.3.303.
3. Albert MS, DeKosky ST, Dickson D, Dubois B, Feldman HH, Fox NC, et al. The diagnosis of mild cognitive impairment due to Alzheimer's disease: recommendations from the National Institute on Aging-Alzheimer's Association workgroups on diagnostic guidelines for Alzheimer's disease. Alzheimer's Dement. 2011;7:270-9. https://doi.org/10.1016/j.jalz.2011.03.008.

4. Donaghy PC, O'Brien JT, Thomas AJ. Prodromal dementia with Lewy bodies. Psychol Med. 2015;45:259-68.

5. Donaghy PC, Taylor JP, O'Brien JT, Barnett N, Olsen K, Colloby SJ, et al. Neuropsychiatric symptoms and cognitive profile in mild cognitive impairment with Lewy bodies. Psychol Med. 2018;48:2384-90.

6. McKeith IG, Ferman TJ, Thomas AJ, Blanc F, Boeve BF, Fujishiro H, et al. Research criteria for the diagnosis of prodromal dementia with Lewy bodies. Neurology. 2020;94(17):743-755.

7. McKeith IG, Boeve BF, Dickson DW, Halliday G, Aarsland D, Attems J, et al. Diagnosis and management of dementia with Lewy bodies fourth consensus report of the DLB Consortium. Neurology. 2017;0:1-13.

8. Thomas AJ, Donaghy P, Roberts G, Colloby SJ, Barnett NA, Petrides G, et al. Diagnostic accuracy of dopaminergic imaging in prodromal dementia with Lewy bodies. Psychol Med. 2019;49:396-402 Available from: https://www. cambridge.org/core/product/identifier/S0033291718000995/type/journal_ article.

9. Delorme A, Makeig S. EEGLAB: an open source toolbox for analysis of single-trial EEG dynamics including independent component analysis. J Neurosci Methods. 2004;134:9-21 Available from: https://linkinghub.elsevier. com/retrieve/pii/S0160791X13000638.

10. Stylianou M, Murphy N, Peraza LR, Graziadio S, Cromarty RA, Killen A, et al. Quantitative electroencephalography as a marker of cognitive fluctuations in dementia with Lewy bodies and an aid to differential diagnosis. Clin Neurophysiol. 2018;129:1209-20 Available from: http://linkinghub.elsevier. com/retrieve/pii/S1388245718302670.

11. Whitham EM, Pope KJ, Fitzgibbon SP, Lewis T, Clark CR, Loveless S, et al. Scalp electrical recording during paralysis: quantitative evidence that EEG frequencies above $20 \mathrm{~Hz}$ are contaminated by EMG. Clin Neurophysiol. 2007; 118:1877-88 Available from: https://linkinghub.elsevier.com/retrieve/pii/S13 88245707001988

12. Bonanni L, Thomas A, Tiraboschi P, Perfetti B, Varanese S, Onofri M. EEG comparisons in early Alzheimer's disease, dementia with Lewy bodies and Parkinson's disease with dementia patients with a 2-year follow-up. Brain. 2008;131:690-705 Available from: http://www.brain.oxfordjournals.org/cgi/ doi/10.1093/brain/awm322

13. Walker MP, Ayre GA, Cummings JL, Wesnes KA, McKeith IG, O'Brien JT, et al. Quantifying fluctuation in dementia with Lewy bodies, Alzheimer's disease, and vascular dementia. Neurol Int. 2000;54:1616-25. https://doi.org/10.1212/ WNL.54.8.1616.

14. Dauwan M, Linszen MMJ, Lemstra AW, Scheltens P, Stam CJ, Sommer IE. EEG-based neurophysiological indicators of hallucinations in Alzheimer's disease: comparison with dementia with Lewy bodies. Neurobiol Aging. 2018;67:75-83 Available from: https://inkinghub.elsevier.com/retrieve/pii/ S0197458018300915

15. Kai T, Asai Y, Sakuma K, Koeda T, Nakashima K. Quantitative electroencephalogram analysis in dementia with Lewy bodies and Alzheimer's disease. J Neurol Sci. 2005;237:89-95 Available from: http:// linkinghub.elsevier.com/retrieve/pii/S0022510X05002005.

16. Babiloni C, Del Percio C, Bordet R, Bourriez JL, Bentivoglio M, Payoux P, et al. Effects of acetylcholinesterase inhibitors and memantine on resting-state electroencephalographic rhythms in Alzheimer's disease patients. Clin Neurophysiol. 2013;124:837-50. https://doi.org/10.1016/j.clinph.2012.09.017

17. Bonanni L, Perfetti B, Bifolchetti S, Taylor J-P, Franciotti R, Parnetti L, et al. Quantitative electroencephalogram utility in predicting conversion of mild cognitive impairment to dementia with Lewy bodies. Neurobiol Aging. 2015;36:434-45 Available from: http://linkinghub.elsevier.com/retrieve/pii/ S0197458014004783.

18. Peraza LR, Cromarty RA, Kobeleva X, Firbank MJ, Killen A, Graziadio S, et al. Electroencephalographic derived network differences in Lewy body dementia compared to Alzheimer's disease patients. Sci Rep. 2018;8:4637 Available from: http://www.nature.com/articles/s41598-018-22984-5.

19. Bonanni L, Franciotti R, Nobili F, Kramberger MG, Taylor JP, Garcia-Ptacek S, et al. EEG markers of dementia with Lewy bodies: a multicenter cohort study. J Alzheimers Dis. 2016;54:1649-57.

20. Cromarty RA, Elder GJ, Graziadio S, Baker M, Bonanni L, Onofrj M, et al. Neurophysiological biomarkers for Lewy body dementias. Clin Neurophysiol. 
2016;127:349-59 Available from: http://linkinghub.elsevier.com/retrieve/pii/ S1388245715006768

21. Babiloni C, Del Percio C, Lizio R, Noce G, Lopez S, Soricelli A, et al. Abnormalities of resting state cortical EEG rhythms in subjects with mild cognitive impairment due to Alzheimer's and Lewy body diseases. J Alzheimer's Dis. 2018;62:247-68 Available from: http://www.medra.org/ servlet/aliasResolver?alias=iospress\&doi=10.3233/JAD-170703.

22. van der Zande JJ, Gouw AA, van Steenoven I, Scheltens P, Stam CJ, Lemstra AW. EEG characteristics of dementia with Lewy bodies, Alzheimer's disease and mixed pathology. Front Aging Neurosci. 2018;10:1-10 Available from: https://www.frontiersin.org/article/10.3389/fnagi.2018.00190/full.

23. Sonnen JA, Montine KS, Quinn JF, Breitner JCS, Montine TJ. Cerebrospinal fluid biomarkers in mild cognitive impairment and dementia. J Alzheimers Dis. 2010;19:301-9.

24. Babiloni C, Del Percio C, Lizio R, Noce G, Lopez S, Soricelli A, et al. Functional cortical source connectivity of resting state electroencephalographic alpha rhythms shows similar abnormalities in patients with mild cognitive impairment due to Alzheimer's and Parkinson's diseases. Clin Neurophysiol. 2018;129:766-82.

\section{Publisher's Note}

Springer Nature remains neutral with regard to jurisdictional claims in published maps and institutional affiliations.

Ready to submit your research? Choose BMC and benefit from:

- fast, convenient online submission

- thorough peer review by experienced researchers in your field

- rapid publication on acceptance

- support for research data, including large and complex data types

- gold Open Access which fosters wider collaboration and increased citations

- maximum visibility for your research: over $100 \mathrm{M}$ website views per year

At $\mathrm{BMC}$, research is always in progress.

Learn more biomedcentral.com/submissions 